\title{
GROUPS IN WHICH EACH ELEMENT COMMUTES WITH ITS ENDOMORPHIC IMAGES
}

\section{R. FAUDREE}

Abstract. Let $G$ be a finite group in which each element commutes with its endomorphic images. We will give a counterexample to the conjecture that $G$ is abelian.

Let $G$ be a finite group in which each element commutes with its endomorphic images. We will give a counterexample to the conjecture that $G$ is abelian.

For $g$ and $h$ in $G, g$ and $g^{-1}\left(h^{-1} g h\right)$ commute. Thus $G$ satisfies the identical relation $(x, y, x)=1$. Therefore $G$ satisfies the identical relations $(x, y, z, w)=1$ and $(x, y, z)^{3}=1$ (see $[1$, p. 322]). Hence $G$ is nilpotent of class $\leqq 3$ and if $G$ is a $p$-group for a prime $p \neq 3, G$ is nilpotent of class $\leqq 2$. We will exhibit for any prime $p$ a nilpotent class $2 p$-group in which each element commutes with all of its endomorphic images.

Let

$$
\begin{array}{r}
G=\left\langle a_{1}, a_{2}, a_{3}, a_{4}: a_{i}^{p 2}=1,\left(a_{i}, a_{j}, a_{k}\right)=1,(1 \leqq i, j, k \leqq 4)\right. \\
\text { and (1) }\left(a_{1}, a_{2}\right)=a_{1}^{p},(2)\left(a_{1}, a_{3}\right)=a_{3}^{p},(3)\left(a_{1}, a_{4}\right)=a_{4}^{p}, \\
\text { (4) } \left.\left(a_{2}, a_{3}\right)=a_{2}^{p},(5)\left(a_{2}, a_{4}\right)=1,(6)\left(a_{3}, a_{4}\right)=a_{3}^{p}\right\rangle .
\end{array}
$$

Let $E(G)$ and $A(G)$ denote the endomorphisms and automorphisms of $G$ respectively. Let $E Z(G)=\{\theta \in E(G): \theta(G) \leqq Z(G)\}$ and $A Z(G)$ $=\left\{\theta \in A(G): \theta(g) g^{-1} \in Z(G)\right.$ for all $\left.g \in G\right\}=$ central automorphisms of $G$.

We will prove

THEOREM. $E(G)=E Z(G) \cup A Z(G)$.

Corollary. Each element in $G$ commutes with its endomorphic images.

Lemma 1. $|G|=p^{8}$.

Proof. Clearly from the defining relations $|G| \leqq p^{8}$. We will construct a group of order $p^{8}$ which satisfies the defining relations of $G$. Let

Received by the editors February 11, 1970 and, in revised form, April 24, 1970.

AMS 1969 subject classifications. Primary 2025, 2040.

Key words and phrases. Finite group, abelian group, endomorphic images, nilpotent class 2 -group. 


$$
\begin{aligned}
& K=\left\langle k_{i}, 1 \leqq i \leqq 4: k_{1}^{p}=k_{3}^{p}=1, k_{2}^{p^{2}}=\right. k_{4}^{p^{2}}=1 \\
&\text { and } \left.\left(k_{i}, k_{j}\right)=1,1 \leqq i, j \leqq 4\right\rangle \\
& \text { and } H=\left\langle h_{1}, h_{3}: h_{1}^{p^{2}}=h_{3}^{p^{2}}=1 \text { and }\left(h_{1}, h_{3}\right)=h_{3}^{p}\right\rangle .
\end{aligned}
$$

$K$ is an abelian group of order $p^{6}$ and $H$ is a nilpotent class 2 group of order $p^{4}$. Let $\sigma_{1}$ and $\sigma_{3}$ be the automorphisms of $K$ determined by $\sigma_{1}\left(k_{1}\right)=k_{1}, \sigma_{1}\left(k_{3}\right)=k_{3}, \sigma_{1}\left(k_{2}\right)=k_{1}^{-1} k_{2}$ and $\sigma_{1}\left(k_{4}\right)=k_{4}^{-p} k_{4}$ and $\sigma_{3}\left(k_{1}\right)=k_{1}$, $\sigma_{3}\left(k_{3}\right)=k_{3}, \sigma_{3}\left(k_{2}\right)=k_{2}^{p} k_{2}$ and $\sigma_{3}\left(k_{4}\right)=k_{3}^{-1} k_{4}$. The subgroup of $A(K)$ generated by $\sigma_{1}$ and $\sigma_{3}$ is an abelian group of exponent $p$ and order $p^{2}$. Let $\theta$ be the homomorphism of $H$ into $A(K)$ determined by $\theta\left(h_{1}\right)$ $=\sigma_{1}$ and $\theta\left(h_{3}\right)=\sigma_{3}$.

Let $S=[K]_{\theta} H=\{k h: h$ in $H$ and $k$ in $K\}$ be the semidirect product of $K$ by $H$ determined by $\theta$. Thus $h k=\left(\theta\left(h^{-1}\right)\right)(k) h . S$ is a group of order $p^{10}$ and the elements $k_{1}^{-1} h_{1}^{p}$ and $k_{3}^{-1} h_{3}^{p}$ generate a subgroup $R$ of order $p^{2}$ in $Z(S)$. Let $T=S / R, t_{1}=h_{1} \cdot R, t_{2}=k_{2} \cdot R, t_{3}=h_{3} \cdot R$ and $t_{4}$ $=k_{4} \cdot R$. The group $T$ of order $p^{8}$ is generated by $\left\{t_{i}: 1 \leqq i \leqq 4\right\}$ and the $t_{i}$ satisfy the defining relations of $G$.

Lemma 2. $Z(G)=G^{\prime}=G^{p}=U_{p}(G)$ where $U_{p}(G)$ is the set of elements whose order divides $p$.

Proof. $G^{\prime}=\left\langle a_{i}^{p}, 1 \leqq i \leqq 4\right\rangle=G^{p}, G^{\prime} \subseteq Z(G)$ and $G^{\prime} \subseteq U_{p}(G)$.

Since $|G|=p^{8},\left|G^{\prime}\right|=p^{4}$. If $[G: Z(G)] \leqq p^{3}$ then $\left|G^{\prime}\right| \leqq p^{3}$ and if $\left[G: U_{p}(G)\right] \leqq p^{3}$ then $\left|G^{p}\right| \leqq p^{3}$.

For a fixed $g \in G$, let

$$
\begin{aligned}
\theta_{0}: G & \rightarrow G \\
h & \rightarrow(h, g) .
\end{aligned}
$$

Since $G$ is nilpotent of class $2, \theta_{\theta}$ is a homomorphism. Ker $\theta_{\theta}=$ $C_{G}(\langle g\rangle)$ and $\left[G: C_{G}(\langle g\rangle)\right]=\left|\theta_{g}(G)\right|$.

LEMma 3. (i) $C_{G}\left(\left\langle a_{1}\right\rangle\right)=G^{\prime} \cdot\left\langle a_{1}\right\rangle$,

(ii) $C_{G}\left(\left\langle a_{2}\right\rangle\right)=C_{G}\left(\left\langle a_{4}\right\rangle\right)=G^{\prime} \cdot\left\langle a_{2}, a_{4}\right\rangle$,

(iii) $C_{G}\left(\left\langle a_{3}\right\rangle\right)=G^{\prime} \cdot\left\langle a_{3}, a_{1} a_{4}\right\rangle$.

Proof. It is easily seen that the right-hand side is contained in the left-hand side. $\theta_{a_{1}}(G)=\left\langle a_{1}^{p}, a_{3}^{p}, a_{4}^{p}\right\rangle$. Thus $\left[G: C_{G}\left(\left\langle a_{1}\right\rangle\right)\right]=p^{3}$, which gives the first equality. Likewise, $\left|\theta_{a_{i}}(G)\right|=p^{2}$ and $\left[G: C_{G}\left(\left\langle a_{i}\right\rangle\right)\right]$ $=p^{2}(2 \leqq i \leqq 4)$.

Lemma 4. Let $n_{i}(1 \leqq i \leqq 4)$ be integers. The matrix 


$$
A=\left(\begin{array}{cccr}
n_{2} & 0 & n_{3} & n_{4} \\
-n_{1} & n_{3} & 0 & 0 \\
0 & -n_{2} & n_{4}-n_{1} & 0 \\
0 & 0 & -n_{3} & -n_{1}
\end{array}\right)
$$

considered over the prime field of characteristic $p$ has rank 0,2 , or 3 .

Proof. Rank $A \neq 4$ since $\operatorname{det} A=0$. One can verify that if all 2 by 2 submatrices of $A$ have determinant $0(\bmod p)$, then $n_{i} \equiv 0(\bmod p)$, $(1 \leqq i \leqq 4)$. Thus Rank $A \neq 1$.

LEMmA 5. If $\theta \notin A(G)$ and $\theta \in E(G)$ then $\theta \in E Z(G)$.

Proof. $\theta \notin A(G)$ implies $\theta(h) \in G^{\prime}$ for some $h \notin G^{\prime}$. Thus $h=$ $\prod_{i=1}^{i} a_{i}^{n_{i}}$ with $n_{i} \neq \equiv 0(\bmod p)$ for at least one $i . \theta\left(a_{i}, h\right)=\left(\theta\left(a_{i}\right), \theta(h)\right)$ $=1(1 \leqq i \leqq 4)$, hence $\left\langle\left(a_{i}, h\right), 1 \leqq i \leqq 4\right\rangle \subseteq \operatorname{Ker} \theta$.

$$
\begin{aligned}
& \left(a_{1}, h\right)=a_{1}^{p n_{2}} a_{3}^{p n_{3}} a_{4}^{p n_{4}}, \\
& \left(a_{2}, h\right)=a_{1}^{-p n_{1}} a_{2}^{p n_{8}}, \\
& \left(a_{3}, h\right)=a_{2}^{-p n_{2}} a_{3}^{p\left(n_{4}-n_{1}\right)}, \\
& \left(a_{4}, h\right)=a_{3}^{-p n_{8}} a_{4}^{-p n_{1}} .
\end{aligned}
$$

The matrix of the powers of $a_{i}^{p}$ in (*) is $A$. Rank $A \geqq 2$ since $n_{i} \not \equiv 0$ $(\bmod p)$ for some $i$. Hence $\left|\operatorname{Ker} \theta \cap G^{\prime}\right| \geqq p^{2}$ and there exists $\left\{h_{i}: 1 \leqq i \leqq 4\right\}$ such that $G=\left\langle h_{1}, h_{2}, h_{3}, h_{4}\right\rangle$ and $\theta\left(h_{1}^{p}\right)=\theta\left(h_{2}^{p}\right)=1$. Thus for $i=1,2, \theta\left(h_{i}\right) \in G^{\prime}$ and $(\theta(G))^{\prime} \subseteq\left\langle\theta\left(h_{3}\right), \theta\left(h_{4}\right)\right\rangle^{\prime}$. Therefore $\left|\theta\left(G^{\prime}\right)\right|$ $=\left|(\theta(G))^{\prime}\right| \leqq p$ and $\left|\operatorname{Ker} \theta \cap G^{\prime}\right| \geqq p^{3}$. We can assume $\theta\left(h_{i}^{p}\right)=1$ and $\theta\left(h_{i}\right) \in G^{\prime}, 1 \leqq i \leqq 3$. Hence $(\theta(G))^{\prime}=\langle 1\rangle, G^{\prime} \subseteq$ Ker $\theta$ and $\theta(G) \subseteq G^{\prime}$. Let

$$
\begin{aligned}
& R=\left\{g \in G:\left[G: C_{G}(\langle g\rangle)\right]=p^{2}\right\}, \\
& R_{1}=\left\{g \in G: g \notin G^{\prime} \text { and } g=a_{2}^{r} a_{4}^{2}\right\}, \\
& R_{2}=\left\{g \in G: g \notin G^{\prime} \text { and } g=a_{1}^{r} a_{3}^{z} a_{4}^{r}\right\} .
\end{aligned}
$$

At least one of the integers $r$ or $s$ is not divisible by $p$ since $g \notin G^{\prime}$.

LEMмA 6. $R=R_{1} \cup R_{2}$.

Proof. Let $h=\prod_{1=1}^{4} a_{i}^{n_{i}} . h \in R$ if and only if $\left|\theta_{h}(G)\right|=p^{2}$. The argument in Lemma 5 yields that $\left|\theta_{h}(G)\right|=p^{2}$ if and only if Rank $A$ $=2$. By inspection one can observe that $\operatorname{Rank} A=2$ if $h \in R_{1} \cup R_{2}$. Let $d_{i j}$ denote the $(i, j)$-minor of $A$. If Rank $A=2$ then $d_{i j} \equiv 0$ $(\bmod p), 1 \leqq i, j \leqq 4$. In particular 


$$
\begin{aligned}
-n_{1}^{2} n_{2} \equiv d_{13} \equiv 0 & (\bmod p) \\
-n_{1}^{2}\left(n_{1}-n_{4}\right) \equiv d_{12} \equiv 0 & (\bmod p) \\
-n_{3}^{2} n_{2} \equiv d_{34} \equiv 0 & (\bmod p) \\
-n_{3} n_{4}\left(n_{1}-n_{4}\right) \equiv d_{41} \equiv 0 & (\bmod p)
\end{aligned}
$$

The equations $(* *)$ imply either $n_{1} \equiv n_{3} \equiv 0(\bmod p)$ or $n_{2} \equiv n_{1}-n_{4}$ $\equiv 0(\bmod p)$.

LeMma 7. $A(G)=A Z(G)$.

Proof. $C_{G}(\langle h\rangle)=G^{\prime} \cdot\left\langle a_{2}, a_{4}\right\rangle$ if $h \in R_{1}$ and $C_{G}(\langle h\rangle)=G^{\prime} \cdot\left\langle a_{3}, a_{1} a_{4}\right\rangle$ if $h \in R_{2}$. Let $\theta \in A(G)$. Either (i) $\theta\left(a_{2}\right), \theta\left(a_{4}\right) \in R_{1}$ and $\theta\left(a_{3}\right) \in R_{2}$ or (ii) $\theta\left(a_{2}\right), \theta\left(a_{4}\right) \in R_{2}$ and $\theta\left(a_{3}\right) \in R_{1}$.

The defining relations 2 and 3 of $G$ imply $\left(\theta\left(a_{1}\right), h_{i}\right)=h_{i}^{p}$ for some $h_{i} \in R_{i}, i=1,2$. Let

$$
\begin{aligned}
& h_{1}=a_{2}^{r} a_{4}^{s}, \\
& h_{2}=a_{1}^{t} a_{3}^{u} a_{4}^{t}, \\
& \theta\left(a_{1}\right)=a_{1}^{\alpha_{1}} a_{2}^{\alpha_{2}} a_{3}^{\alpha_{3}} a_{4}^{\alpha_{4}} .
\end{aligned}
$$

Therefore we get, among other equalities, the following:

$r \alpha_{1} \equiv r\left(\alpha_{3}+1\right) \equiv s \alpha_{3} \equiv s\left(\alpha_{1}-1\right) \equiv\left(\alpha_{1}-\alpha_{4}-1\right) u \equiv\left(\alpha_{1}-\alpha_{4}-1\right) t \equiv 0(\bmod p)$.

The only solutions are $\alpha_{1}-1 \equiv \alpha_{3} \equiv \alpha_{4}(\bmod p)$.

Consider case (ii). Thus

$$
\begin{array}{ll}
\theta\left(a_{1}\right)=a_{1}^{\alpha_{1}} a_{2}^{\alpha_{2}} a_{3}^{\alpha_{1}-1} a_{4}^{\alpha_{1}-1,} & \theta\left(a_{2}\right)=a_{1}^{\beta_{1}} a_{3}^{\beta_{2}} a_{4}^{\beta_{1}}, \\
\theta\left(a_{3}\right)=a_{2}^{\gamma_{2}} a_{4}^{\gamma_{4}}, & \theta\left(a_{4}\right)=a_{1}^{\delta_{1}} a_{3}^{\delta_{2}} a_{4}^{\delta_{1}} .
\end{array}
$$

From the defining relations 1,4 and 6 of $G$ we get, among other equalities, the following:

$$
\begin{aligned}
\alpha_{1}+\alpha_{2} \beta_{1} & \equiv \alpha_{1}-\beta_{1}-1 \equiv \beta_{1}\left(\gamma_{2}-1\right) \equiv \beta_{1}\left(\gamma_{4}-1\right) \\
& \equiv \beta_{3}\left(\gamma_{4}-1\right) \equiv \delta_{3} \gamma_{4} \equiv\left(\delta_{3}-1\right) \gamma_{2} \equiv 0 \quad(\bmod p) .
\end{aligned}
$$

Either $\beta_{1} \neq 0(\bmod p)$ or $\beta_{3} \neq \equiv(\bmod p)$. Hence $\gamma_{4}-1 \equiv \delta_{3} \equiv \gamma_{2} \equiv \beta_{1}$ $\equiv \alpha_{1} \equiv \alpha_{1}-1 \equiv 0(\bmod p)$, a contradiction.

Consider case (i). Thus

$$
\begin{array}{ll}
\theta\left(a_{1}\right)=a_{1}^{\alpha_{1}} a_{2}^{\alpha_{2}} a_{3}^{\alpha_{1}-1} a_{4}^{\alpha_{1}-1}, & \theta\left(a_{2}\right)=a_{2}^{\beta_{2} a_{4}^{\beta_{4}}}, \\
\theta\left(a_{3}\right)=a_{1}^{\gamma_{1}} a_{3}^{\gamma_{3}} a_{4}^{\gamma_{1}}, & \theta\left(a_{4}\right)=a_{2}^{\delta_{2}} a_{4}^{\delta_{4}} .
\end{array}
$$

From the defining relations 1,4 and 6 of $G$ we get, among other equalities, the following: 


$$
\begin{array}{r}
\alpha_{1}\left(\beta_{2}-1\right) \equiv\left(\alpha_{1}-1\right) \beta_{2}+\alpha_{2} \equiv \alpha_{1}\left(\beta_{4}-1\right)+1 \equiv \beta_{2} \gamma_{1} \equiv \beta_{2}\left(\gamma_{3}-1\right) \\
\equiv \beta_{4} \gamma_{3} \equiv \beta_{4}\left(\gamma_{1}+1\right) \equiv \delta_{2}\left(\gamma_{1}-1\right) \equiv\left(\delta_{4}-1\right) \gamma_{3} \equiv 0 \\
(\bmod p) .
\end{array}
$$

If $\beta_{4} \neq \equiv 0(\bmod p)$, then $\gamma_{3} \equiv \gamma_{1}+1 \equiv \beta_{2} \equiv \alpha_{1} \equiv 0(\bmod p)$. Thus $1 \equiv 0(\bmod p)$, a contradiction. If $\beta_{4} \equiv 0(\bmod p)$, then $\beta_{2} \neq 0(\bmod p)$ and

$$
\gamma_{1} \equiv \gamma_{3}-1 \equiv \delta_{2} \equiv \delta_{4}-1 \equiv \alpha_{1}-1 \equiv \alpha_{2} \equiv \beta_{2}-1 \equiv 0 \quad(\bmod p) .
$$

Hence $\theta \in A Z(G)$.

\section{REFERENCES}

1. M. Hall, Jr., The theory of groups, Macmillan, New York, 1959. MR 21 \#1996.

UNIVERSITY OF ILLINOIS, URBANA, ILLINOIS 61801 